\title{
Effects of Mindful Acceptance and Reappraisal Training on Maladaptive Beliefs About Rumination
}

\author{
Shian-Ling Keng ${ }^{1}$ - Moria J. Smoski ${ }^{2,3}$ • Clive J. Robins ${ }^{2,3}$
}

Published online: 8 January 2016

(C) Springer Science+Business Media New York 2016

\begin{abstract}
The present study examined the relative effects of mindful acceptance and reappraisal on metacognitive attitudes and beliefs in relation to rumination and negative experiences. A small but growing literature has compared the effects of these strategies on immediate emotional experience, but little work has examined the broader, metacognitive impact of these strategies, such as maladaptive beliefs about rumination. One hundred and twenty-nine participants who reported elevated symptoms of depression were randomly assigned to receive brief training in mindful acceptance, reappraisal, or no training prior to undergoing an autobiographical sad mood induction. Participants rated their beliefs in relation to rumination and negative experiences before and after instructions to engage in mood regulation. Results showed that relative to reappraisal or no training, training in mindful acceptance resulted in greater decreases in maladaptive beliefs about rumination. The study suggests that training in mindful acceptance promotes beneficial changes in metacognitive attitudes and beliefs relevant to depression, and contributes to a greater understanding of the mechanisms through which mindfulness-based interventions lead to positive outcomes.
\end{abstract}

Electronic supplementary material The online version of this article (doi:10.1007/s12671-015-0480-x) contains supplementary material, which is available to authorized users.

Shian-Ling Keng

psykeng@nus.edu.sg

1 Department of Psychology, National University of Singapore, Block AS4, \#02-22, 9 Arts Link, Singapore 117570, Singapore

2 Department of Psychiatry and Behavioral Sciences, Duke University Medical Center, Box 3026, Durham, NC, USA

3 Department of Psychology and Neuroscience, Duke University, Durham, NC, USA
Keywords Emotion regulation · Mindfulness $\cdot$ Acceptance . Reappraisal · Depression

\section{Introduction}

It is widely known that rumination increases vulnerability to depression (Nolen-Hoeksema and Morrow 1991; Teasdale and Barnard 1993). Individuals who ruminate tend to engage in repetitive negative thinking about themselves, their behaviors, their life situations, and their ability to cope. Beyond rumination, metacognition and attitudes related to rumination and negative experiences have been shown to be important etiological factors in depression (Papageorgiou and Wells 2001, 2003). Metacognition refers to the aspect of the information processing system that monitors and exerts control over thoughts and emotions (Singer and Dobson 2007). One aspect of metacognition concerns metacognitive appraisals that one makes in the context of rumination and negative thoughts (Papageorgiou and Wells 2003). For example, one may engage in "positive" metacognitive appraisals about rumination, i.e., thinking that rumination can help solve one's problems, or negative metacognitive appraisals about rumination, i.e., believing that negative thoughts are harmful and to be avoided. While much work has demonstrated the role of these metacognitive processes in the context of depression, less is known regarding the extent to which these processes can be modified, especially in a laboratory context.

Reappraisal involves reformulating the meaning or interpretation of an emotion-inducing situation to reduce its emotional impact (Gross 1998). It has been conceptualized as an antecedent-focused strategy, which refers to attempts to regulate emotional tendencies at or prior to the onset of emotions. When used as an antecedent-focused strategy, with instructions to engage in reappraisal prior to the start of an 
emotion-inducing stimulus (e.g., prior to watching an emotional film), reappraisal is found to be more effective than suppression of emotion expression (Gross 1998), rumination (Grisham, Flower, Williams, and Moulds 2009), and distraction (McRae et al. 2010) in reducing distress. However, when used as an "online" regulation strategy, with instructions to regulate provided after an emotional response has already begun (e.g., in the midst of watching an emotional film), reappraisal is less effective than distraction at reducing sadness (Sheppes and Meiran 2007) and results in greater sympathetic nervous system activation (Sheppes, Catran, and Meiran 2009). Initiating reappraisal late as opposed to early (antecedent-focused reappraisal) in an emotional situation may pose greater self-control challenges presumably because it requires individuals to override strong, well-established negative interpretations of the situation, as suggested by a study showing that online reappraisal resulted in impaired cognitive performance (Sheppes and Meiran 2008).

Mindfulness has been defined as the awareness that arises through "paying attention in a particular way: on purpose, in the present moment, and non-judgmentally" (Kabat-Zinn 1994, p. 4). Bishop et al. (2004) proposed that mindfulness encompasses two components: self-regulation of attention and adoption of an attitude of curiosity, non-judgment, and acceptance towards one's experiences. These aspects of mindfulness have been regarded as potentially effective antidotes against psychological distress, which often involves maladaptive tendencies to avoid, suppress, or over-engage with distressing thoughts and emotions (Hayes and Feldman 2004). Experimental studies examining the effects of mindfulness on emotion functioning have used various instructions to create laboratory analogue of a state of mindfulness or a mindful (or accepting) way of relating to one's experiences. These instructions include acceptance (i.e., instructions to accept one's thoughts and emotions as they are, which is one key component of mindfulness), focused attention (i.e., instructions to focus one's attention on an object-typically one's breath - a commonly used meditative approach to induce a state of mindfulness), or a combination of focused attention and instructions to accept one's thoughts and emotions as they are (which we termed "mindful acceptance" in this paper). Focused attention has been found to be more effective than rumination, distraction, or worry inductions in lowering negative mood (Arch and Craske 2006; Broderick 2005) in undergraduate samples. Among individuals with mood or anxiety disorders, instructions to accept one's experiences as they are (which also included a rationale to accept one's experiences) have been found to result in lower negative affect and decreased heart rate in response to emotional film clips, as compared to suppression (Campbell-Sills et al. 2006). Mindful acceptance, on the other hand, has been found to be more effective than rumination in reducing negative affect among previously depressed (Singer and Dobson 2007) and currently depressed individuals (Huffziger and Kuehner 2009).

A small number of studies have directly compared the effectiveness of reappraisal and acceptance. Studies have demonstrated a benefit for both acceptance and reappraisal (used as an antecedent-focused strategy) in downregulating subjective and physiological indicators of negative affect (Hofmann et al. 2009; Wolgast et al. 2011), with some studies finding a benefit for reappraisal over acceptance (Hofmann et al. 2009; Szasz et al. 2011). Notably, the type of acceptance training provided in the latter studies involved instructions to simply accept one's thoughts and emotions, without including a rationale to the instructions. It remains to be examined empirically whether the simplified nature of the instructions may have accounted for the reduced efficacy of acceptance instructions in these studies.

Moving beyond the effects of mindful acceptance and reappraisal on immediate emotional experience, it is important to focus on the broader emotional and metacognitive impact of these strategies, such as metacognitive beliefs about rumination and negative experiences. Comparison of these two strategies in particular is of interest as both strategies involve responding to one's thoughts and emotions, but in distinctive ways. Whereas mindfulness-based strategies emphasize changing the relationship to one's thoughts and emotions by viewing them as metacognitive events and attending to them non-judgmentally (Brown et al. 2007), cognitive changebased strategies such as reappraisal emphasize evaluating and changing the content of one's thoughts and emotions (Hofmann and Asmundson 2008). These two classes of emotion regulation strategies mirror key intervention techniques that are emphasized in mindfulness- and acceptance-based therapies, such as mindfulness-based cognitive therapy (Segal et al. 2002), and traditional cognitive behavioral therapies, such as cognitive therapy (Beck et al. 1979), respectively. Examination of the strategies therefore has implications for understanding the mechanisms through which these therapies work. Because mindfulness and reappraisal emphasize different ways of relating to internal experiences, they may differentially impact one's metacognitive styles and beliefs.

Singer and Dobson (2007) distinguished among three types of metacognitive styles. Positive metacognitive appraisal about rumination (or positive beliefs about rumination) refers to the belief that repeated thinking or ruminating about problems is beneficial and can lead to solution of the problems (Papageorgiou and Wells 2001). Negative metacognitive appraisal about rumination (or negative beliefs about rumination) refers to belief in the uncontrollability and harmfulness of rumination, which might lead to attempts to distract from or avoid negative thoughts and feelings. Both types of metacognitive appraisals are positively associated with rumination and depression in non-clinical samples and clinically depressed individuals (Papageorgiou and Wells 2001, 2003). 
Negative metacognitive appraisals about rumination have also been found to predict, prospectively, higher levels of depression in a non-clinical sample (Papageorgiou and Wells 2009). According to the Wells and Matthews' Self-Regulatory Executive Function (S-REF) model of emotional disorders, an important etiological factor in depression is rumination, which is maintained by beliefs that rumination is helpful (Papageorgiou and Wells 2003). The negative consequences resulting from rumination in turn lead to appraisals of rumination as uncontrollable and harmful, which further contribute to the experience of depression. An alternative to engaging in positive or negative metacognitive appraisals about rumination is maintaining an attitude of acceptance of, or openness towards negative, experiences, with the understanding that thoughts and emotions are simply passing events in the mind (Teasdale et al. 1995). Singer and Dobson (2007) suggested that increased acceptance towards negative thoughts and feelings may facilitate disengagement from a ruminative thinking style.

Training in mindfulness, or aspects of mindfulness (such as acceptance and focused attention), may lead to beneficial changes in the above-stated metacognitive styles. Singer and Dobson (2007) found that, relative to rumination and distraction, a brief training in mindful acceptance significantly lowered negative beliefs about rumination in a remitted depressed sample. Instructions involving focused attention on breathing have also been found to result in increased acceptance towards negative experiences, as reflected by greater willingness to remain in contact with aversive visual stimuli, compared with unfocused attention (Arch and Craske 2006). Other research has found that acceptance instructions (with rationale included) resulted in reduced behavioral avoidance (Eifert and Heffner 2003) and greater willingness to participate in a second carbon dioxide challenge (Levitt et al. 2004) compared with suppression or no instructions. Taken together, these studies suggest that acceptance, mindful acceptance, or focused attention training may foster more adaptive metacognitive styles by decreasing maladaptive beliefs about rumination or increasing acceptance of negative experiences. Training in reappraisal, on the other hand, may not result in such changes given that the training emphasizes the need to change one's internal experiences, which assumes the perspective that negative internal experiences are aversive and, therefore, needs to be altered in some way. To the best of our knowledge, no study has yet examined the effects of mindful acceptance on beliefs about rumination and attitudes towards negative experiences relative to those of reappraisal. This study aimed to examine the hypotheses that mindful acceptance, relative to reappraisal and no instruction, would result in significantly greater decreases in positive and negative beliefs about rumination, and greater increases in acceptance of negative experiences, in a sample of individuals with elevated symptoms of depression.

\section{Method}

\section{Participants}

The sample and procedure of this study have been described in detail in Keng et al. (2013), which reported on the emotional and cognitive sequelae of reappraisal and mindful acceptance. Because the outcome variables in this study are psychopathological processes relevant to depression, we recruited individuals with elevated depressive symptoms. Participants were eligible if they were between 18 and 55 years old and obtained a score between 10 and 29 on the Beck Depression Inventory (BDI; Beck et al. 1979), which was administered online as a screening instrument in the recruitment process. Due to ethical concerns (as the study involved a negative mood induction component), participants were excluded from the study if they scored above 29 on the BDI or endorsed suicidal ideation, defined by a score of 3 on the suicidality item of the BDI. A total of 129 participants were recruited and randomly assigned in blocks of three to receive training in mindful acceptance $(n=43)$ and reappraisal $(n=43)$ or to a no-instruction condition ( $n=43)$. Random assignment was accomplished using an online random number generator (randomizer.org). The allocation of assignments was concealed using a password-protected document accessible only by a research assistant not involved in enrolling participants. All participants were recruited from an undergraduate research subject pool and from the community, and received credits towards a course research requirement or US\$20, respectively, for their participation. Of the sample, $55.4 \%$ were student participants. This study was approved by Duke University's Institutional Review Board.

\section{Procedure}

Upon providing informed consent, participants completed several self-report questionnaires (see below section), which included a demographic data form, the BDI, and the Attitudes towards Negative Experiences Scale (ATNES). Participants randomized to the reappraisal or mindful acceptance conditions received standardized verbal instructions in their assigned strategies (see Fig. 1). The instructions for the mindful acceptance condition, adapted from Singer and Dobson (2007), emphasized accepting thoughts and emotions as they are without judging them (acceptance) and included a focused attention experiential exercise (see Appendix A). Instructions for the reappraisal condition were adapted from Grisham et al. (2009) and Ray et al. (2008) (see Appendix B). Participants were trained to reframe the meaning of an emotional event to reduce its emotional impact and engaged in an exercise involving reappraising a hypothetical situation. At the end of the 10-min training session, participants rated the perceived 
Fig. 1 Study flowchart

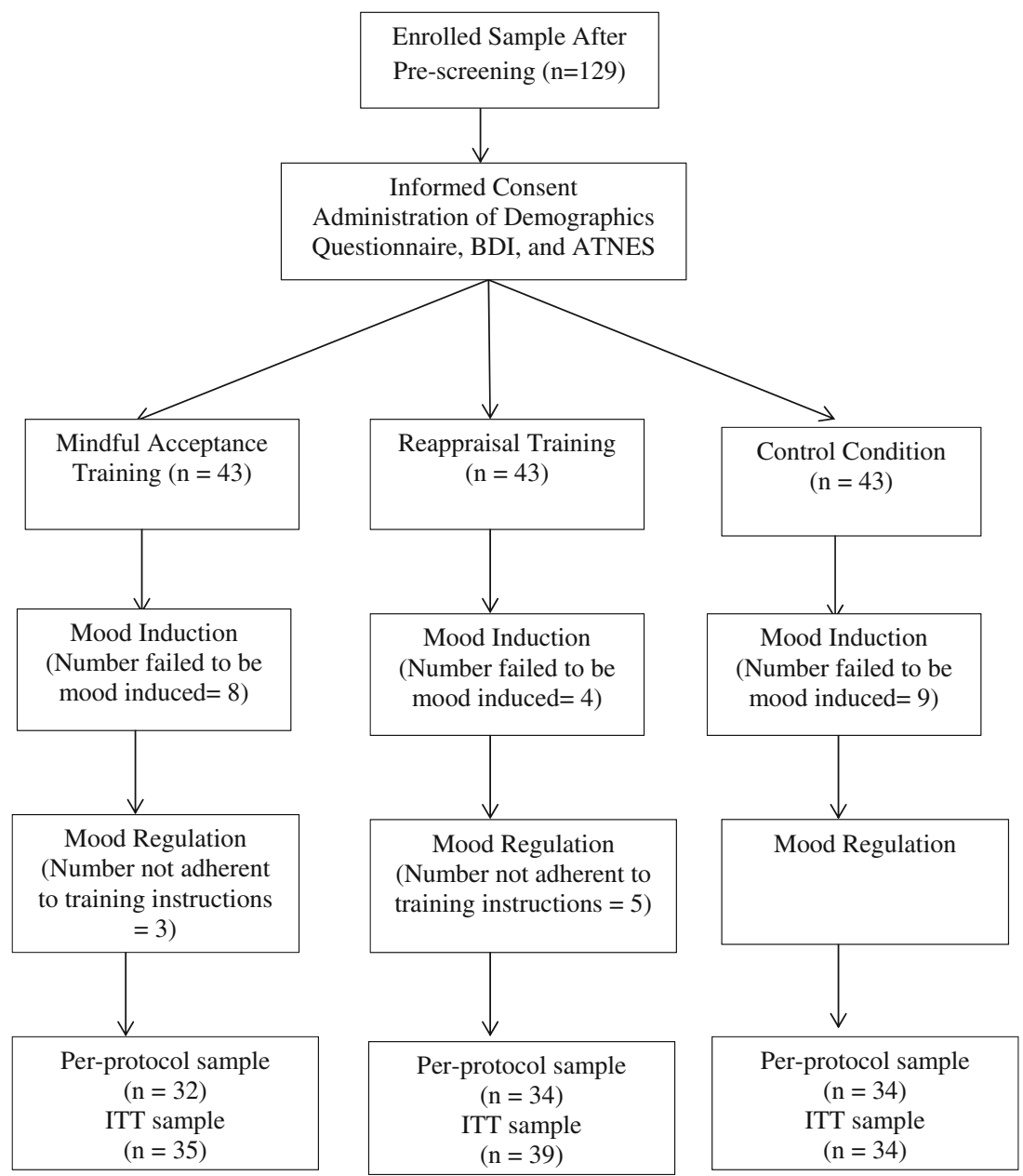

usefulness of their assigned technique. Participants assigned to the no-instruction condition received no training.

Participants then underwent a 10 -min mood induction procedure that involved simultaneous negative autobiographical recall (write and think about three events that made them feel lonely, sad, rejected, or hurt) and mood-suggestive music ("Adagio-G Minor" composed by Albinoni, played at half speed). Participants rated their mood (level of sadness) on a 10-cm visual analogue scale (VAS) at pre- and post-mood induction. Following induction, participants who were assigned to the mindful acceptance or reappraisal condition received instructions to apply their assigned strategy. Participants in the no-training condition were instructed to simply "respond to their mood." All participants rated their mood on the VAS every $30 \mathrm{~s}$ during the mood regulation period, which lasted $5 \mathrm{~min}$. At the end of the mood induction and regulation period, participants rated the extent to which they engaged in several emotion regulation strategies (mindful acceptance, reappraisal, distraction, rumination, and suppression) during the regulation period on a 7-point Likert-type scale and completed the ATNES again. Participants also rated the level of perceived credibility and enthusiasm of the experimenter. At the end of the experiment, all participants completed a color-word Stroop task. Results associated with the task and participants' sad mood ratings were reported in Keng et al. (2013).

\section{Measures}

Demographics The demographic data form contained questions about participants' age, gender, ethnicity, education background, income, history of receiving psychological and/ or psychiatric treatment, and prior experience with mindfulness training.

Depressive Symptoms The BDI (Beck et al. 1979) was administered at baseline to assess symptoms of depression. Traditional classifications of BDI scores are none or minimal depression (0-9), mild to moderate depression, (10-18), moderate to severe depression (19-29), and severe depression (3063). The scale's internal consistency in this sample was 0.82 .

Beliefs About Rumination and Acceptance Towards Negative Experiences The ATNES (Singer and Dobson 2007) was 
administered at baseline and after the mood regulation period to assess state changes in beliefs about rumination and level of acceptance towards negative experiences. It is a 15 -item questionnaire that consists of three subscales: (1) positive attitude, which refers to the belief that rumination is a useful coping strategy (e.g., "When feelings of depression arise, dwelling on those feelings help to make sense of them."), (2) negative attitude, which refers to the belief that rumination is harmful and uncontrollable, resulting in attempts to voluntarily control thinking (e.g., "When something bad happens, I try to avoid and control the depressive thoughts that come to mind."), and (3) acceptance, which refers to an attitude of acceptance and openness towards negative experiences (e. g., Remaining mindful of the present moment is helpful in reducing depressing feelings."). Items are rated on a 5 -point scale ( $1=$ strongly disagree; $4=$ strongly agree). The scale's internal consistency in the current sample was 0.80 for positive attitude, 0.70 for negative attitude, and 0.56 for acceptance.

\section{Data Analyses}

All analyses were conducted using SPSS. First, following Keng et al. (2013), participants who reported a mood shift of less than 1 point ( $1 \mathrm{~cm}$ on a $10-\mathrm{cm}$ line) were considered nonresponders to the mood induction procedure and excluded from the subsequent analyses. Participants assigned to the mindfulness and reappraisal conditions who reported less than a minimum score of 4 on a 7-point scale on their respective manipulation check question were considered non-adherent to the instructions and also excluded from subsequent analyses. Between-group differences in use of emotion regulation strategies (mindfulness, reappraisal, distraction, suppression, and rumination) during the regulation period were evaluated in a one-way multivariate analysis of variance (MANOVA). Baseline characteristics of participants across the three study conditions were compared using a one-way MANOVA on all continuous variables and chi-square tests for categorical variables. For any variable for which there was a significant difference across conditions, a correlation was calculated between the variable and each of the dependent measures. If the analysis produced significant findings, the variable was included as a covariate in subsequent analyses. A one-way MANOVA was also conducted to examine if there were differences among groups on their ratings of the enthusiasm and credibility of the experimenter. An independent samples $t$ test was also conducted to compare perceived usefulness of the randomized emotion regulation strategy in the mindfulness group and the reappraisal group.

In terms of the primary analyses, due to pre-test differences in each of the outcome variables, change score analysis (oneway analysis of variance (ANOVA) on change scores), instead of analysis of covariance (ANCOVA), was used to compare changes in positive beliefs about rumination, negative beliefs about rumination, and acceptance of negative experiences from pre- to post-regulation across the three study conditions. According to Weinfurt (2000), ANCOVA addresses the question of whether there are significant between-group differences at post-test, provided that groups have the same mean scores at pre-test. Given that the groups in the present study differed in their mean scores for each of the outcome variables at baseline, change score analysis would be the more valid analytic approach (Weinfurt 2000). This analysis addresses the question of whether the three groups demonstrated different amounts of change from pre- to post-regulation. Because the data from this study have been used in a previously published study (Keng et al. 2013), resulting in a total of five dependent variables being tested (including those in this study), we applied the Benjamini and Hochberg procedure Benjamini and Hochberg (1995) to correct for experimentwise error inflation. We first conducted the primary analyses using the per-protocol sample $(n=100)$ and repeated the analyses using the intent-to-treat (ITT) sample $(n=108)$, which included participants who failed to engaged in the mindful acceptance or reappraisal condition.

\section{Results}

\section{Mood Induction and Strategy Implementation Manipulation} Check Twenty-one participants $(16.3 \%)$ did not meet the criterion for successful mood induction. There were also no group differences in the effect of the mood induction on sadness.

Participants assigned to the mindful acceptance and reappraisal conditions who reported a minimum score of 4 on a 7-point scale on their respective manipulation check question (which inquired the extent to which they implemented their assigned strategy) were considered adherent to the instructions and included in the analyses. Eight (9\%) participants assigned to the mindful acceptance or reappraisal condition failed to adequately engage in the technique to which they were assigned. This leaves a per-protocol sample size of 100 (reappraisal $n=34$; acceptance $n=32$, noinstruction $n=34$ ).

Between-group differences in use of emotion regulation strategies (mindful acceptance, reappraisal, distraction, suppression, and rumination) during the regulation period were evaluated in a one-way MANOVA. There was a significant effect of group on self-reported use of emotion regulation strategies during the regulation period, $F(12$, $186)=5.08, p<.001$. Follow-up univariate ANOVAs revealed significant between-group differences in the extent to which participants engaged in mindful acceptance $(p<.001)$ and reappraisal $(p<.001)$. As expected, the 
mindful acceptance group reported significantly greater engagement in mindful acceptance $(M=5.59)$ than the reappraisal group $(M=4.74 ; p=.006)$, and the control group $(M=4.12 ; p<.001)$, and the reappraisal group engaged in reappraisal $(M=5.56)$ to a significantly greater extent than the mindful acceptance group $(M=3.81 ; p<.001)$, and the control group $(M=3.62 ; p<.001)$. Within the control group, there was no statistically significant difference in the degree to which participants engaged in reappraisal versus mindful acceptance. There were no significant between-group differences in suppression, positive distraction, distraction, or rumination during the regulation period.

\section{Analyses of Pre-test Differences Across Conditions Table 1} presents demographic and clinical characteristics of the three groups. There were no group differences on any of the categorical baseline variables. Using Pillai's trace, there was a significant effect of group on the set of continuous variables at baseline $(F(22,176)=1.99$, $p<.01)$. Follow-up analyses showed that there were significant between-group differences at baseline in positive beliefs about rumination $(p<.01)$, negative beliefs about rumination $(p<.05)$, and acceptance of negative experiences $(p<.01)$. Post-hoc comparisons using the Bonferroni test indicated that positive beliefs about rumination were significantly higher in the mindful acceptance group $(p<.01)$ and the reappraisal group $(p<.05)$ than in the control group. Similarly, acceptance of negative experiences was significantly higher in the mindful acceptance group $(p<.05)$ and the reappraisal group $(p<.01)$ compared to the control group. Relative to the control group, the mindful acceptance group also reported significantly higher negative beliefs about rumination $(p<.05)$.

There were no significant differences between experimental groups on perceived levels of enthusiasm and credibility of the experimenter. The mindful acceptance and reappraisal groups did not differ significantly on perceived usefulness of their assigned technique.

Effects of Condition on Acceptance Towards Negative Experiences and Beliefs About Rumination Table 2 shows the descriptive and test statistics of changes in positive beliefs about rumination, negative beliefs about rumination, and acceptance of negative experiences across the three groups. Using the per-protocol sample, one-way ANOVA on changes in positive beliefs about rumination revealed a significant between-group effect, $F(2,97)=3.35, p=.04, \eta^{2}=.06$. Post-hoc contrasts using Fisher's LSD test showed that the decreases were significantly greater in the mindful acceptance group than in the control group (mean difference $=2.09$, $\mathrm{SE}=.86$ ),$p=.02$, Cohen's $d=.56$. There were no significant
Table 1 Sample characteristics across conditions (per-protocol sample)

\begin{tabular}{lllll}
\hline & $\begin{array}{l}\text { All participants } \\
(n=100)\end{array}$ & $\begin{array}{l}\text { Mindful } \\
\text { acceptance } \\
(n=32)\end{array}$ & $\begin{array}{l}\text { Reappraisal } \\
(n=34)\end{array}$ & $\begin{array}{l}\text { Control } \\
(n=34)\end{array}$ \\
\hline Variable & & & & \\
Female (\%) & 69.00 & 68.80 & 67.60 & 70.60 \\
Caucasian (\%) & 53.00 & 53.10 & 50.00 & 44. \\
Married/cohabiting (\%) & 27.00 & 25.00 & 38.20 & 17.60 \\
Employed (\%) & 45.00 & 46.90 & 41.20 & 47.10 \\
Currently in therapy (\%) & 12.00 & 9.40 & 17.60 & 8.80 \\
Previously in therapy (\%) & 43.00 & 46.90 & 44.10 & 38.20 \\
Taking psychotropic & 19.00 & 21.90 & 14.70 & 20.60 \\
$\quad$ medications (\%) & 34.00 & 37.50 & 35.30 & 29.40 \\
Having taken psychotropic medications (\%) & 49.00 & 53.13 & 58.82 & 35.29 \\
Education (\% with at least & & & & $M(\mathrm{SD})$ \\
$\quad$ a college degree) & $M(\mathrm{SD})$ & $M(\mathrm{SD})$ & $M(\mathrm{SD})$ & $26.53(9.83)$ \\
Age & $29.04(11.49)$ & $30.28(12.47)$ & $30.38(11.98)$ & $16.03(5.59)$ \\
BDI & $16.05(4.98)$ & $16.50(4.91)$ & $15.65(4.50)$ & $12.59(3.38)$ \\
ATNES-POS & $14.42(4.00)$ & $15.72(4.07)$ & $15.03(3.93)$ & $15.18(4.09)$ \\
ATNES-NEG & $15.95(3.50)$ & $17.28(2.85)$ & $15.47(3.13)$ & $14.79(3.71)$ \\
ATNES-ACC & $16.31(3.46)$ & $16.88(3.15)$ & $17.29(3.04)$ & 14.79 \\
\hline
\end{tabular}

BDI Beck Depression Inventory, ATNES-POS Attitude towards Negative Experiences Scale-Positive Beliefs about Rumination Subscale, ATNES-NEG Attitude towards Negative Experiences Scale-Negative Beliefs about Rumination Subscale, ATNES-ACC Attitude towards Negative Experiences Scale-Acceptance of Negative Experiences Subscale 
Table 2 Descriptive and test statistics of changes in beliefs about rumination and acceptance towards negative experiences across conditions (per-protocol sample)

\begin{tabular}{lllll}
\hline & $\begin{array}{l}\text { Mindful acceptance } \\
M(\mathrm{SD})\end{array}$ & $\begin{array}{l}\text { Reappraisal } \\
M(\mathrm{SD})\end{array}$ & $\begin{array}{l}\text { Control } \\
M(\mathrm{SD})\end{array}$ & Test statistics \\
\hline $\begin{array}{c}\text { Change in positive beliefs about } \\
\text { rumination }\end{array}$ & $-.47(3.40)^{\mathrm{a}}$ & $-.03(2.91)$ & $1.62(4.02)^{\mathrm{a}}$ & $F(2,97)=3.35, p=.04$ \\
$\begin{array}{c}\text { Change in negative beliefs about } \\
\text { rumination }\end{array}$ & $-1.44(3.03)^{\mathrm{b}, \mathrm{c}}$ & $2.06(2.80)^{\mathrm{b}}$ & $1.79(3.43)^{\mathrm{c}}$ & $F(2,97)=12.91, p<.001$ \\
$\begin{array}{c}\text { Change in acceptance of negative } \\
\text { experiences }\end{array}$ & $1.81(2.58)^{\mathrm{d}}$ & $.32(1.87)^{\mathrm{d}, \mathrm{e}}$ & $1.85(4.00)^{\mathrm{e}}$ & $F(2,59.73)=4.51$, \\
& & $p=.02$
\end{tabular}

Change scores were calculated by subtracting scores obtained at baseline (pre-mood induction) from scores obtained at post-regulation

${ }^{a}$ Decreases in the mindful acceptance condition were significantly greater than in the control condition, $p<.05$

${ }^{\mathrm{b}}$ Decreases in the mindful acceptance condition were significantly greater than in the reappraisal condition, $p<.001$

${ }^{\mathrm{c}}$ Decreases in the mindful acceptance condition were significantly greater than in the control condition, $p<.001$

${ }^{\mathrm{d}}$ Increases in the mindful acceptance condition were significantly greater than in the reappraisal condition, $p<.05$

${ }^{\mathrm{e}}$ Increases in the control condition were significantly greater than in the reappraisal condition, $p<.05$ differences between the mindful acceptance group and reappraisal group, $p=.61$, Cohen's $d=.14$. There was a trend for greater increase in positive beliefs about rumination in the control group versus the reappraisal group, $p=.05$, Cohen's $d=.47$. Groups also differed significantly on changes in negative beliefs about rumination, $F(2$, $97)=12.91, p<.001, \eta^{2}=.21$, with post-hoc analysis showing that decreases were significantly larger in the mindful acceptance group than the reappraisal group (mean difference $=3.50, \mathrm{SE}=.76), p<.001$, Cohen's $d=1.20$, and the control group (mean difference $=3.23, \mathrm{SE}=.76$ ), $p<.001$, Cohen's $d=1.00$. There was no significant difference between the reappraisal group and the control group on this variable, $p=.73$, Cohen's $d=.09$.

Lastly, the Welch test (used in place of one-way ANOVA because of heterogeneity of variance in the dependent variable) indicated a significant between-group effect on acceptance of negative experiences, $F(2,59.73), p=.02, \eta^{2}=.06$. Post-hoc contrasts showed that the mindful acceptance group demonstrated significantly greater increases in acceptance of negative experiences than the reappraisal group (mean difference $=1.49, \mathrm{SE}=.56), p<.05$, Cohen's $d=.66$. There was no significant difference between the mindfulness acceptance group and the control group on this variable, $p=.73$, Cohen's $d=.01$. Interestingly, the control group demonstrated significantly greater increases in acceptance compared to the reappraisal group (mean difference $=1.52, \mathrm{SE}=.72$ ),$p=.04$, Cohen's $d=.49$. The findings related to variable however should be interpreted with caution given that the corresponding subscale demonstrated weak internal consistency $(\alpha=.56)$. All the findings above remained significant after applying the Benjamini-Hochberg correction. We repeated the analyses using the ITT sample and replicated the above findings, with the exception that the trend for greater increases in positive beliefs about rumination in the control group versus the reappraisal group in the post-hoc analysis became statistically significant, $p=-.03$, Cohen's $d=.52$.

\section{Discussion}

Using an experimental paradigm, the present study showed that brief training in mindful acceptance resulted in significantly greater decreases in negative beliefs about rumination relative to reappraisal, in a sample of participants reporting elevated symptoms of depression. Compared to no instruction, training in mindful acceptance also resulted in significantly greater decreases in both positive and negative beliefs about rumination. Mindful acceptance was also associated with greater increases in acceptance of negative experiences compared to reappraisal, although this finding should be regarded as tentative, given the low internal consistency associated with the corresponding subscale.

The finding that training in mindful acceptance resulted in significantly greater decreases in negative beliefs about rumination compared to reappraisal and no instruction is consistent with the findings by Singer and Dobson (2007). However, unlike Singer and Dobson, the study also demonstrated greater reductions in positive beliefs about rumination in the mindful acceptance group relative to the control conditions. Variations in sample characteristics (e.g., higher depression severity in this sample vs. Singer and Dobson's sample) and/ or other methodological factors may have contributed to the discrepancy in findings. The results suggest that a brief experiential training in mindful acceptance may lower, at least temporarily, both positive and negative beliefs about rumination. These variables have been theoretically linked to the etiology of depression and empirically demonstrated to be 
maladaptive beliefs that correlate positively with depression in non-clinical and clinically depressed adults (Papageorgiou and Wells 2001, 2003). The findings underscore the utility of mindfulness in modifying maladaptive cognitive styles and beliefs that maintain rumination and depression (Papageorgiou and Wells 2003). They further suggest that modification of metacognitive styles and beliefs may be an important mechanism of change for the effects of established mindfulness-based interventions (Hofmann et al. 2010).

The finding that mindful acceptance training resulted in increases in acceptance towards negative experiences relative to reappraisal supports a theoretical work proposing that mindfulness facilitates emotional acceptance (Hayes et al. 1999; Segal et al. 2002) and contributes to a growing empirical literature emphasizing the role of mindfulness in increasing willingness to tolerate negative emotions (e.g., Arch and Craske 2006; Eifert and Heffner 2003; Levitt et al. 2004). Despite being tentative, the finding is consistent with the theoretical basis of acceptance-based interventions (such as acceptance and commitment therapy), which aim to increase acceptance of one's experiences and reduce the extent to which behaviors are regulated by aversive thoughts and emotions (Hayes 2008). Notably however, there was no significant difference in increases in acceptance between the mindful acceptance and the no-instruction conditions. The fact that we did not include a detailed assessment of the types of strategies that each participant engaged in in this condition makes it difficult to determine potential reasons underlying the lack of a difference. It is plausible that the lack of active regulation in the no-instruction condition (which arguably overlaps with a mindful approach) may have contributed to an increase in acceptance of negative experiences, but future research needs to examine this hypothesis using more precise assessments.

Overall, the findings of this study are consistent with theoretical predictions regarding the mechanisms through which mindfulness-based interventions treat depression, one of which is that mindfulness training alleviates symptoms of depression through reducing maladaptive rumination (Teasdale et al. 1995; Heeren and Philippot 2011; Shahar et al. 2010). The present study further highlighted the role of shifts in metacognitive beliefs about rumination as an additional mechanism of change underlying the effects of mindfulness training. Unfortunately, the fact that the present study did not assess rumination per se precluded further analyses that could illustrate the temporal dynamics between changes in beliefs about rumination vs. rumination. Based on Wells and Matthews' Self-Regulatory Executive Function (S-REF) model of emotional disorders, which postulates that ruminative thinking is perpetuated by unhelpful beliefs about rumination, we predict that improvements in maladaptive beliefs would precede changes in rumination (Papageorgiou, and Wells 2003). However, it is plausible too that reduced rumination due to mindfulness training may lead to experiential insight that facilitates changes in metacognitive beliefs about rumination (i.e., as one experiences the benefits of ruminating less, one may let go of previously held unhelpful beliefs about rumination). Future research should examine closely the relationship between metacognitive beliefs about rumination and ruminative thinking and how mindfulness training may alter these two processes simultaneously.

This study can be noted for several strengths, such as use of a randomized controlled experimental design and control for experimenter effects and participants' adherence to manipulation instructions. The sample is also racially and ethnically diverse: Approximately half of the sample was Caucasian (53\%), followed by Asian (22\%), African American $(15 \%)$, American Indian (6\%), and Hispanic (4\%). There are several limitations to the study. One limitation of the study is its reliance on self-report methods to assess attitudes towards negative experiences. Future research should employ additional modes of assessments (e.g., behavioral measures) to strengthen the validity of the findings. Similarly, assessing adherence to training instructions through self-report has limited validity. Additionally, we administered the ATNES, the only known measure that assesses metacognitive styles in the context of depressive experiences at the time of the study. Our analysis showed that the internal consistencies for the negative beliefs and acceptance subscales were less than ideal $(0.70$ and 0.56 , respectively). Use of measures with more established validity and reliability would have enhanced our findings. It is also unknown the extent to which the results of this laboratory study are generalizable to coping with negative situations in daily life. The study employed autobiographical recall of personally relevant memories to induce sad mood; however, in real life, people encounter a variety of (both personally relevant and non-relevant) stress-inducing situations. The effects of mindful acceptance and reappraisal may therefore vary by the nature and severity of stressors encountered. The training instructions provided in the study also serve only as rough approximations of how processes relevant to mindfulness and reappraisal are taught in the context of psychotherapy, although effort was made to make the training instructions as comprehensive as possible (e.g., by incorporating an experiential/practice component in the training instructions for both conditions) within the time limit of the experiment session. Further, the study recruited participants based on selfreported depression symptoms (individuals reporting mild to moderate levels of depressive symptoms who may or may not meet full criteria for clinical depression), which limits the generalizability of the results to individuals meeting diagnostic criteria for major depression. It is notable, though, that the present sample had an average BDI score of 16, which exceeds the threshold for probable clinical depression developed via ROC analyses (Lasa et al. 2000).

The present study points to several worthwhile directions for future research. Future studies should continue to examine 
psychological processes that are commonly and differentially impacted by mindful acceptance and reappraisal, as such research would help elucidate the mechanisms through which these strategies, as well as interventions that employ these strategies as key elements, impact psychological functioning. Also, although the study identified changes in beliefs about rumination as outcomes unique to mindful acceptance, it is unknown to what extent these changes account for known clinical effects of mindfulness training, such as reduced risk of depressive relapses (Ma and Teasdale 2004; Teasdale et al. 2000). An intervention trial with temporal assessments of hypothesized outcome and mediating variables will help shed light on these questions. For instance, future research could examine the effects of an established mindfulness-based intervention program (e.g., mindfulness-based stress reduction) on metacognitive styles and associated changes in clinical outcomes through the use of global self-report, behavioral, performance-based, and/or ecological momentary assessment methods. Not only will such research enable researchers to examine metacognitive processes in the context of real life comprehensively it will also provide important insight into how these processes relate to relevant clinical outcomes.

\section{Appendices}

\section{Appendix A}

Mindfulness Training Instructions

Now I am going to train you on how to use a strategy for dealing with negative emotions. We call this technique "mindfulness."

What this technique involves is to simply be aware of your thoughts, emotions, and experiences in a non-judgmental manner, to accept them to be as they are in the present moment, without engaging in thinking about them or pushing them away.

Typically, our natural tendency when experiencing difficult thoughts and emotions is that we tend to think about them over and over again, judge them as good or bad, or try to push them away and not want to deal with them. The technique of mindfulness is to avoid these two opposites; so instead of engaging with our thoughts and emotions or pushing them away, we practice accepting and being aware of them as simply thoughts and emotions and watching them come and go, as if they are waves in the ocean (or clouds against the sky).

One good way of understanding what mindfulness is is via doing an experiential exercise.

Now, close your eyes, if that feels comfortable for you, and allow the body to relax... (PAUSE)... the first step is being aware, really aware, of what is going on with you right now. (PAUSE).... Notice if there are any physical sensations in the body... tightness... or tension in any part of the body... (PAUSE)... any thoughts you are having... (PAUSE)... any emotions you are feeling... (PAUSE)... When you are ready, I would like you to tell me what are you noticing now about your present experience...... (PAUSE)........ What physical sensations are you feeling? (PAUSE).......... What emotions are you feeling? (PAUSE)........ What thoughts are running through your mind (PAUSE) ..........

If you like, you can think of thoughts as if they were projected on the screen at the cinema. You sit, watching the screen, waiting for the thoughts or images to arise. When they do, you pay attention to them so long as they are there "on the screen" and then let them go as they pass away.

If you like, you can acknowledge the presence of these thoughts and images, perhaps saying "Ah, there you are, that's how it is right now" without judging them as good or bad. And similarly with sensations in the body "Are there sensations of tension, of holding or whatever?" And again, awareness of them, simply noting them. 'OK, that is how it is right now.'

[The experimenter emphasizes non-judgment and acceptance of the experiences noted in the exercise.]

To summarize, the purpose of this technique is to bring your attention back to your direct, present experience, rather than engaging in thinking about them (or what they might mean) or turning your attention away from them. The idea is to notice and allow your present experience, including your thoughts and emotions, in a non-judgmental manner.

\section{Appendix B}

\section{Reappraisal Training Instructions}

Now I am going to train you on how to use a strategy for dealing with negative emotions. We call this technique "reappraisal."

What this technique involves is to change our thoughts or interpretations about events that upset us - meaning - to change the way we think about the event.

Typically, the reason we experience difficult thoughts and emotions is that we tend to have very negative interpretations about the event that triggers them. The technique of reappraisal is to change the way we interpret the event, so that we feel less negatively about it. It is the attempt to develop a more positive interpretation about a situation that at first glance may appear very negative to us.

There are many ways one can change one's interpretations about a situation. One can start, for example, by asking "Is this the only way I can think about the event or the situation?" "Is there a more positive way of construing the event so that I feel less negatively about it?" "What could be another way to look at what happened?" If you like, you can also challenge your negative thoughts or interpretations of an event, disprove them 
by reasoning or by drawing from your other experiences, and replace them with more positive interpretations.

Let us do a practice exercise to demonstrate how this technique works. Let us say you have a colleague, with whom you have a fairly good relationship, who would always smile at, or say hi to, you each time you run into her. However, there is this one time when you ran into her and she was not smiling at you. What might be some thoughts that you have about that in that moment?

[At this point, the experimenter works with the participant to come up with various possible interpretations and demonstrate how each interpretation might lead to a different emotional experience. Finally, the experimenter works with the participant to come up with an interpretation that will lead to less negative positive emotions.]

As you can see, adopting a different interpretation of a negative situation might change how we feel about it. Another way to reappraise an event is by thinking about the event from a perspective other than one's own, say an impartial observer or a third person. For example, one can ask: "If a friend of mine were to face a similar situation, what would I tell him or her? What sort of advice would I give him or her?"

Also, one can try to think of some positive aspects of the event, such as lessons that you have learned and ways you can improve in the future if the same event or situation was to occur again. Think of whether there is any positive meaning that you can derive from the incident.

To summarize, the purpose of this technique is to think about the situation that upsets us in a different way, or cast it in more positive light, so that we are less emotionally impacted by it.

\section{References}

Arch, J. J., \& Craske, M. G. (2006). Mechanisms of mindfulness: emotion regulation following a focused breathing induction. Behaviour Research and Therapy, 44, 1849-1858.

Beck, A. T., Rush, A. J., Shaw, B. F., \& Emery, G. (1979). Cognitive therapy of depression. New York: Guilford.

Benjamini, Y., \& Hochberg, Y. (1995). Controlling the false discovery rate: a practical and powerful approach to multiple testing. Journal of the Royal Statistical Society: Series B Methodological, 57(1), 289-300.

Bishop, S. R., Lau, M., Shapiro, S., Carlson, L., Anderson, N. D., Carmody, J., et al. (2004). Mindfulness: a proposed operational definition. Clinical Psychology: Science and Practice, 11, 230-241.

Broderick, P. C. (2005). Mindfulness and coping with dysphoric mood: contrasts with rumination and distraction. Cognitive Therapy and Research, 29, 501-510.

Brown, K. W., Ryan, R. M., \& Creswell, J. D. (2007). Mindfulness: theoretical foundations and evidence for its salutary effects. Psychological Inquiry, 18(4), 211-237.

Campbell-Sills, L., Barlow, D. H., Brown, T. A., \& Hofmann, S. G. (2006). Effects of suppression and acceptance on emotional responses of individuals with anxiety and mood disorders. Behaviour Research and Therapy, 44, 1251-1263.
Eifert, G. H., \& Heffner, M. (2003). The effects of acceptance versus control contexts on avoidance of panic-related symptoms. Journal of Behavior Therapy and Experimental Psychiatry, 34, 293-312.

Grisham, J., Flower, K., Williams, A., \& Moulds, M. (2009). Reappraisal and rumination during recall of a sad memory. Cognitive Therapy and Research, 35, 1-8.

Gross, J. J. (1998). Antecedent- and response-focused emotion regulation: divergent consequences for experience, expression, and physiology. Journal of Personality and Social Psychology, 74, 224-237.

Hayes, S. C. (2008). Climbing our hills: a beginning conversation on the comparison of acceptance and commitment therapy and traditional cognitive behavioral therapy. Clinical Psychology: Science and Practice, 15, 286-295.

Hayes, A. M., \& Feldman, G. (2004). Clarifying the construct of mindfulness in the context of emotion regulation and the process of change in therapy. Clinical Psychology: Science and Practice, $11(3), 255-262$.

Hayes, S. C., Strosahl, K. D., \& Wilson, K. G. (1999). Acceptance and commitment therapy: an experiential approach to behavior change. New York: Guilford Press.

Heeren, A., \& Philippot, P. (2011). Changes in ruminative thinking mediate the clinical benefits of mindfulness: preliminary findings. Mindfulness, 2(1), 8-13.

Hofmann, S. G., \& Asmundson, G. J. (2008). Acceptance and mindfulness-based therapy: new wave or old hat? Clinical Psychology Review, 28(1), 1-16.

Hofmann, S. G., Heering, S., Sawyer, A. T., \& Asnaani, A. (2009). How to handle anxiety: the effects of reappraisal, acceptance, and suppression strategies on anxious arousal. Behaviour Research and Therapy, 47, 389-394.

Hofmann, S. G., Sawyer, A. T., Witt, A. A., \& Oh, D. (2010). The effect of mindfulness-based therapy on anxiety and depression: a metaanalytic review. Journal of Consulting and Clinical Psychology, $78,169-183$.

Huffziger, S., \& Kuehner, C. (2009). Rumination, distraction, and mindful self-focus in depressed patients. Behaviour Research and Therapy, 47, 224-230.

Kabat-Zinn, J. (1994). Wherever you go there you are: mindfulness meditation in everyday life. New York: Hyperion.

Keng, S. L., Robins, C. J., Smoski, M. J., Dagenbach, J., \& Leary, M. R. (2013). Reappraisal and mindfulness: a comparison of subjective effects and cognitive costs. Behaviour Research and Therapy, 51(12), 899-904.

Lasa, L., Ayuso-Mateos, J. L., Vazquez-Barquero, J. L., Diez-Manrique, F. J., \& Dowrick, C. F. (2000). The use of the Beck Depression Inventory to screen for depression in the general population: a preliminary analysis. Journal of Affective Disorders, 57(1), 261-265.

Levitt, J. T., Brown, T. A., Orsillo, S. M., \& Barlow, D. H. (2004). The effects of acceptance versus suppression of emotion on subjective and psychophysiological response to carbon dioxide challenge in patients with panic disorder. Behavior Therapy, 35, 747-766.

Ma, S. H., \& Teasdale, J. D. (2004). Mindfulness-based cognitive therapy for depression: replication and exploration of differential relapse prevention effects. Journal of Consulting and Clinical Psychology, $72(1), 31-40$.

McRae, K., Hughes, B., Chopra, S., Gabrieli, J. D., Gross, J. J., \& Ochsner, K. N. (2010). The neural bases of distraction and reappraisal. Journal of Cognitive Neuroscience, 22(2), 248-262.

Nolen-Hoeksema, S., \& Morrow, J. (1991). A prospective study of depression and posttraumatic stress symptoms after a natural disaster: the 1989 Loma Prieta earthquake. Journal of Personality and Social Psychology, 61(1), 115-121.

Papageorgiou, C., \& Wells, A. (2001). Positive beliefs about depressive rumination: development and preliminary validation of a self-report scale. Behavior Therapy, 32, 13-26. 
Papageorgiou, C., \& Wells, A. (2003). An empirical test of a clinical metacognitive model of rumination and depression. Cognitive Therapy and Research, 27, 261-273.

Papageorgiou, C., \& Wells, A. (2009). A prospective test of the clinical metacognitive model of rumination and depression. International Journal of Cognitive Therapy, 2(2), 123-131.

Ray, R. D., Wilhelm, F., \& Gross, J. (2008). All in the mind's eye? Anger rumination and reappraisal. Journal of Personality and Social Psychology, 94, 133-145.

Segal, Z. V., Williams, J. M. G., \& Teasdale, J. D. (2002). Mindfulnessbased cognitive therapy for depression: a new approach to preventing relapse. New York, NY: Guilford Press.

Shahar, B., Britton, W. B., Sbarra, D. A., Figueredo, A. J., \& Bootzin, R. R. (2010). Mechanisms of change in mindfulness-based cognitive therapy for depression: preliminary evidence from a randomized controlled trial. International Journal of Cognitive Therapy, 3(4), 402-418.

Sheppes, G., \& Meiran, N. (2007). Better late than never? On the dynamics of online regulation of sadness using distraction and cognitive reappraisal. Personality and Social Psychology Bulletin, 33, 15181532.

Sheppes, G., \& Meiran, N. (2008). Divergent cognitive costs for online forms of reappraisal and distraction. Emotion, 8, 870-874.

Sheppes, G., Catran, E., \& Meiran, N. (2009). Reappraisal (but not distraction) is going to make you sweat: physiological evidence for self-control effort. International Journal of Psychophysiology, 71, 91-96.

Singer, A. R., \& Dobson, K. S. (2007). An experimental investigation of the cognitive vulnerability to depression. Behaviour Research and Therapy, 45, 563-575.

Szasz, P. L., Szentagotai, A., \& Hofmann, S. G. (2011). The effect of emotion regulation strategies on anger. Behaviour Research and Therapy, 49, 114-119.

Teasdale, J. D., \& Barnard, P. J. (1993). Affect, cognition, and change: remodelling depressive thought. Hove: Erlbaum.

Teasdale, J. D., Segal, Z., \& Williams, J. M. (1995). How does cognitive therapy prevent depressive relapse and why should attentional control training help? Behaviour Research and Therapy, 33, 25-39.

Teasdale, J. D., Segal, Z. V., Williams, J. M. G., Ridgeway, V. A., Soulsby, J. M., \& Lau, M. A. (2000). Prevention of relapse/ recurrence in major depression by mindfulness-based cognitive therapy. Journal of Consulting and Clinical Psychology, 68, 615-623.

Weinfurt, K. P. (2000). Repeated measures analyses: ANOVA, MANOVA, and HLM. In L. G. Grimm \& P. R. Yarnold (Eds.), Reading and understanding more multivariate statistics. Washington: American Psychological Association.

Wolgast, M., Lundh, L.-G., \& Viborg, G. (2011). Cognitive reappraisal and acceptance: an experimental comparison of two emotion regulation strategies. Behaviour Research and Therapy, 49, 858-866. 